\title{
APPROXIMATE ISOMETRIES IN BOUNDED SPACES
}

\author{
R. L. SWAIN
}

Approximate isometries have been studied in a paper by $\mathrm{D}$. H. Hyers and S. M. Ulam. ${ }^{1}$ By an 6 -isometry, they mean a transformation $T$ of a metric space $M$ into another such that for each pair $x, y$ of points of $M$

$$
|\rho(x, y)-\rho(T(x), T(y))|<\epsilon,
$$

where $\rho(a, b)$ means the distance between the points $a$ and $b$. Their Theorem 4 shows that if $E$ is a finite-dimensional Euclidean space or a real Hilbert space, then for any $\epsilon$-isometry of $E$ into itself, there is an actual isometry of $E$ into itself such that the distance between the images of any point under these two transformations is less than $10 \epsilon$. Their treatment does not concern bounded spaces.

The purpose of this paper is to show that the type of $k$ uniformity which Hyers and Ulam obtained in their complete spaces cannot be obtained in general even in very special bounded spaces, but that an $\eta, \epsilon$ type relation holds for any compact metric space.

False Proposition. ${ }^{2}$ If $E$ is a bounded domain in the plane, there is a positive integer $k$ such that if $M$ is any (convex) subset of $E$ and $T$ is an $\epsilon$-isometry of $M$ into a subset of the plane, there exists an isometry $U$ of $M$ such that for each point $P$ of $M$,

$$
\rho(T(P), U(P))<k \epsilon .
$$

Example. Suppose that the proposition is true. Let $E$ be the interior of the circle with center $(0,0)$ and radius 2 . Let $M$ consist of the triangle $A B C$ together with its interior (or let $M$ be any subset of this triangular region which contains the points $A, B, C)$, where $A=(-1,0), B=(1,0), C=(0, a)$, with $a=1 / 4 k$. Let $T$ be the transformation which throws each point $(x, y)$ of $M$ into the point $(x, y+a(1-|x|))$. Suppose that $\left(x_{1}, y_{1}\right)$ and $\left(x_{2}, y_{2}\right)$ are any two 1950.

Presented to the Society, November 28, 1942; received by the editors November 9 ,

${ }^{1}$ D. H. Hyers and S. M. Ulam, On approximate isometries, Bull. Amer. Math. Soc. vol. 51 (1945) pp. 288-292.

2 The author suggests that the following proposition may be true: There is a number $k$ such that if $M$ is any convex subset of the plane and $T$ is any $\epsilon$-isometry of $M$ (with $T(M)$ also in the plane), then there exists an isometry $U$ of $M$ such that if $P$ is any point of $M, \rho(T(P), U(P))<k \epsilon / \omega$. Here $\omega$ means the relative thickness of $M$, which could be defined as the ratio of the diameter of the largest circular disk contained in $M$ to the diameter of $M$. 
points of $M$. Then

$$
\begin{aligned}
& \begin{aligned}
\Delta= & \left|\rho\left(T\left(x_{2}, y_{2}\right), T\left(x_{1}, y_{1}\right)\right)-\rho\left(\left(x_{2}, y_{2}\right),\left(x_{1}, y_{1}\right)\right)\right| \\
= & \mid\left\{\left[\left(y_{2}-y_{1}\right)-a\left(\left|x_{2}\right|-\left|x_{1}\right|\right)\right]^{2}+\left(x_{2}-x_{1}\right)^{2}\right\} 1 / 2 \\
& -\left\{\left(y_{2}-y_{1}\right)^{2}+\left(x_{2}-x_{1}\right)^{2}\right\}^{1 / 2} \mid=
\end{aligned} \\
& \left|\frac{-2 a\left(y_{2}-y_{1}\right)+a^{2}\left(\left|x_{2}\right|-\left|x_{1}\right|\right)}{\left\{\frac{\left[\left(y_{2}-y_{1}\right)-a\left(\left|x_{2}\right|-\left|x_{1}\right|\right)\right]^{2}+\left(x_{2}-x_{1}\right)^{2}}{\left(\left|x_{2}\right|-\left|x_{1}\right|\right)^{2}}\right\}^{1 / 2}+\left\{\frac{\left(y_{2}-y_{1}\right)^{2}+\left(x_{2}-x_{1}\right)^{2}}{\left(\left|x_{2}\right|-\left|x_{1}\right|\right)^{2}}\right\}^{1 / 2}}\right| \\
& \quad \leqq \frac{2 a \cdot a+a^{2}}{2}=\frac{3 a^{2}}{2}, \\
& \text { since }\left|y_{2}-y_{1}\right| \leqq a,|| x_{2}|-| x_{1}|| \leqq 1, \text { and }\left(x_{2}-x_{1}\right)^{2} \geqq\left(\left|x_{2}\right|-\left|x_{1}\right|\right)^{2} \text {. (Note } \\
& \text { that if } \left.\left|x_{1}\right|=\left|x_{2}\right|, \Delta=0 .\right) \text { With } \epsilon=2 a^{2}, \text { then, } T \text { is an } \epsilon \text {-isometry of } M . \\
& \text { Then } k \epsilon=a / 2 . \text { Hence } U(A) \text { and } U(B) \text { lie within the circles with radii } \\
& a / 2 \text { and with centers at } T(A)=A \text { and } T(B)=B \text {, respectively, } \\
& \text { and the ordinate of } U(C) \text { exceeds } 3 a / 2 \text {. Hence the altitude from } \\
& U(C) \text { of the triangle } U(M) \text { exceeds } a, \text { which is a contradiction. }
\end{aligned}
$$

Theorem. Suppose that $M$ is a subset of a compact metric space $S$ and that $\eta$ is a positive number. Then there exists a positive number $\epsilon$ such that if $T$ is any $\epsilon$-isometry of $M$ into a subset of $S$, there exists an isometry $U$ of $M$ such that for each point $x$ of $M, \rho(T(x), U(x))<\eta$.

Proof. Suppose that the statement of the theorem does not hold for a given $S, M$, and $\eta$. It follows that for each positive integer $n$, there exists a $1 / n$-isometry $V_{n}$ of $M$ such that if $V$ is any isometry of $M$, there is some point $x$ of $M$ such that $\rho\left(V_{n}(x), V(x)\right) \geqq \eta$. Since $M$ is separable, there exists a sequence $\left\{x_{i}\right\}$ of distinct points of $M$ such that $\sum x_{i}$ is dense in $M$. Since $S$ is compact, there exists a sequence $\{r(1 n)\}$ of positive integers such that the sequence $\left\{V_{r(1 n)}\left(x_{1}\right)\right\}$ converges to a point $y_{1}$ of $S$. Again $\{r(1 n)\}$ contains a subsequence $\{r(2 n)\}$ such that $\left\{V_{r(2 n)}\left(x_{2}\right)\right\}$ converges to a point $y_{2}$. This process continues. Then for each positive integer $k(n>k)$, $\{r(n n)\}$ is a subsequence of $\{r(k n)\}$, so that for each $i,\left\{V_{r(n n)}\left(x_{i}\right)\right\}$ converges to a point $y_{i}$. For each $n$, denote $V_{r(n n)}$ by $T_{n}$. For each point $x$ of $M$, let $U(x)$ be a definite point which is the sequential limit point of some subsequence of $\left\{T_{n}(x)\right\}$. Then $U$ is a one-to-one transformation of $M$.

If we now consider only the sequences $\left\{T_{n}\left(x_{i}\right)\right\}$, each converging to $y_{i}=U\left(x_{i}\right)$, it is easy to see that since $T_{n}$ is $1 / n$-isometric, $U$ is an isometry of $\sum x_{i}$ into $\sum y_{i}$. Then, since $\sum x_{i}$ is dense in $M$, it is not difficult (but tedious) to show that $T_{n}(x)$ converges to $U(x)$ for 
each point $x$ of $M$, and that $U$ is an isometry of $M$. It remains to be shown that for some $n$, and for all $x$ of $M, \rho\left(T_{n}(x), U(x)\right)<\eta$.

For each point $x$ of $M$, let $R_{x}$ be an open sphere with radius $\eta / 4$ and center $U(x)$. The collection of these open spheres covers the closure of $U(M)$, hence contains a finite sub-collection $G$ covering $U(M)$, no two elements of $G$ being identical. Enumerate the elements of $G: R_{1}, R_{2}, \cdots, R_{k}$. For each $i(1 \leqq i \leqq k)$, let $z_{i}$ be the point of $M$ such that $U\left(z_{i}\right)$ is the center of $R_{i}$. It is now easy to set up a sequence of inequalities involving $x, z_{m}, T_{n}(x), T_{n}\left(z_{m}\right), U(x)$, to show that there exists a number $j$ such that if $n>j$, then if $x$ is any point of $M, R_{m}$ containing $U(x), \rho\left(T_{n}(x), U(x)\right)<\eta$. From this contradiction, the theorem follows. ${ }^{3}$

Suppose that we desire to determine the shape of a physical body. We measure distances within the body, and from the set of measurements construct an empirical model of it, the measured figures being reproduced therein, within the error of measurement. The model is thus a spatial "map" of the body. We then attempt by rigid motion to bring the "map" into close conjunction with the body. The results of this paper tell us the following:

(1) Though we assign a maximum error of measurement $e$, there will not necessarily be a natural number $k$ such that for any body, its "map" can be brought to within ke of it, throughout.

(2) But given any positive number $e$, then for any given body, there will be a natural number $k$ such that the "map" can be brought to within $e$ of the body provided that we keep our error of measurement less than $e / k$.

State University of New York,

State Teachers College, New Paltz, N. Y.

${ }^{3} \mathrm{~T}$. Rado has pointed out to me that in the case of continuous e-isometries, the proof of the theorem may be obtained by means of the following sequence of lemmas, each classical or trivial: (1) Given $T_{n}$ a continuous $1 / n$-isometry, then $\left\{T_{n}\right\}$ is equi-continuous, (2) Given $\left\{T_{n}\right\}$ equi-continuous, then $\left\{T_{n}\right\}$ contains a uniformly convergent subsequence, (3) Given $T_{n}$ a continuous $1 / n$-isometry, and $T_{n} \rightarrow T$ uniformly, then $T$ is an isometry. 